\title{
ON THE EXISTENCE OF UNI-INSTANTANEOUS $Q$-PROCESSES WITH A GIVEN FINITE $\mu$-INVARIANT MEASURE
}

\author{
BRENTON GRAY, ${ }^{* * *}$ \\ PHIL POLLETT $* * * *$ AND \\ HANJUN ZHANG, ${ }^{* * * * *}$ University of Queensland
}

\begin{abstract}
Let $S$ be a countable set and let $Q=\left(q_{i j}, i, j \in S\right)$ be a conservative $q$-matrix over $S$ with a single instantaneous state $b$. Suppose that we are given a real number $\mu \geq 0$ and a strictly positive probability measure $m=\left(m_{j}, j \in S\right)$ such that $\sum_{i \in S} m_{i} q_{i j}=-\mu m_{j}$, $j \neq b$. We prove that there exists a $Q$-process $P(t)=\left(p_{i j}(t), i, j \in S\right)$ for which $m$ is a $\mu$-invariant measure, that is $\sum_{i \in S} m_{i} p_{i j}(t)=\mathrm{e}^{-\mu t} m_{j}, j \in S$. We illustrate our results with reference to the Kolmogorov 'K1' chain and a birth-death process with catastrophes and instantaneous resurrection.
\end{abstract}

Keywords: Markov chain; $q$-matrix; birth-death process; construction theory

2000 Mathematics Subject Classification: Primary 60J27

Secondary $60 \mathrm{~J} 35$

\section{Introduction}

We begin with a conservative $q$-matrix over a countable set $S$; that is, a collection $Q=$ $\left(q_{i j}, i, j \in S\right)$ of real numbers that satisfy $0 \leq q_{i j}<\infty, i, j \in S, j \neq i ; q_{i}:=-q_{i i} \leq \infty$, $i \in S$; and $\sum_{j \neq i} q_{i j}=q_{i}, i \in S$.

We shall assume that $Q$ has a single instantaneous state; that is, a state $b \in S$ such that $q_{b}=\infty$ and $q_{i}<\infty$ for $i \neq b$. A set of real-valued functions $P(t)=\left(p_{i j}(t), i, j \in S\right)$ defined on $(0, \infty)$ is called a standard transition function or process if

$$
\begin{array}{rlrl}
p_{i j}(t) & \geq 0, & & i, j \in S, t>0, \\
\sum_{j \in S} p_{i j}(t) & \leq 1, & i \in S, t>0, \\
p_{i j}(s+t) & =\sum_{k \in S} p_{i k}(s) p_{k j}(t), & & i, j \in S, s, t>0, \\
\lim _{t \downarrow 0} p_{i j}(t) & =\delta_{i j}, & & i, j \in S,
\end{array}
$$

where $\delta_{i j}$ is the Kroneker delta. The process $P$ is then honest if equality holds in (2) for some (and, thus, all) $t>0$, and it is called a $Q$-transition function (or $Q$-process) if $p_{i j}^{\prime}(0+)=q_{i j}$ for each $i, j \in S$.

Received 1 July 2003; revision received 30 March 2005.

* Postal address: Department of Mathematics, University of Queensland, Queensland 4072, Australia.

** Email address: bgray@ pacificsolutions.com.au

*** Email address: pkp@maths.uq.edu.au

**** Email address: hjz@maths.uq.edu.au 
If $\mu$ is some fixed nonnegative real number, a collection of strictly positive numbers $m=$ $\left(m_{j}, j \in S\right.$ ) is called a $\mu$-subinvariant measure (on $S$ ) for $Q$ if $\sum_{i \in S} m_{i} q_{i j} \leq-\mu m_{j}, j \in S$, and is called $\mu$-invariant if

$$
\sum_{i \in S} m_{i} q_{i j}=-\mu m_{j}, \quad j \in S
$$

Here, we shall suppose that $m$ is a finite measure (i.e. $\sum_{i \in S} m_{i}<\infty$ ) which is almost $\mu$-invariant for $Q$, that is

$$
\sum_{i \in S} m_{i} q_{i j}=-\mu m_{j}, \quad j \neq b,
$$

and we will show that there always exists a $Q$-process $P$ such that $m$ is a $\mu$-invariant measure (on $S$ ) for $P$, that is

$$
\sum_{i \in S} m_{i} p_{i j}(t)=\mathrm{e}^{-\mu t} m_{j}, \quad j \in S, t>0
$$

(When $\mu=0$, all of the above notions reduce to the more common ones of invariance and subinvariance.) Note that if we were given a $\mu$-invariant measure $m$ for a particular $Q$-process $P$, then, since (7) may be rewritten as

$$
\sum_{i \neq j} m_{i} p_{i j}(t)+\left(1-\mathrm{e}^{-\mu t}\right) m_{j}=\left(1-p_{j j}(t)\right) m_{j},
$$

Fatou's lemma would give

$$
\sum_{i \neq j} m_{i} q_{i j}+\mu m_{j} \leq q_{j} m_{j}
$$

for all $j \in S$, meaning that $m$ would be $\mu$-subinvariant for $Q$. However, under what conditions is $m \mu$-invariant for $Q$ ? In Section 2, we provide necessary and sufficient conditions for $m$ to be almost invariant for $Q$ and delay addressing the interesting question of whether or not $\sum_{i \neq b} m_{i} q_{i b}=\infty$, which would be the remaining requirement for (5) to hold; this question will be considered in Section 6.

Here, we are assuming that $Q$ is uni-instantaneous. When $Q$ is totally stable, that is $q_{i}<\infty$ for all $i \in S$, the relationship between (5) and (7) is well understood, and has been divined completely for the minimal $Q$-process $F$. It was shown by Tweedie [14] that if $m$ is a $\mu$-invariant measure for $F$, then it is also $\mu$-invariant for $Q$. Conversely [8], [9], if $m$ is $\mu$-invariant for $Q$, then it is $\mu$-subinvariant for $F$ and $\mu$-invariant for $F$ if and only if the equations

$$
\sum_{i \in S} y_{i} q_{i j}=-v y_{j}, \quad 0 \leq y_{j} \leq m_{j}, j \in S,
$$

have only the trivial solution for some (and, thus, all) $v<\mu$. This result holds whether or not $S$ is irreducible and does not require $m$ to be finite. If, as we are assuming here, $m$ is finite, then, for $\mu$ to be strictly positive, it is necessary that $F$ be dishonest. Furthermore, if $F$ is the unique $Q$-process satisfying the forward equations, then $m$ is $\mu$-invariant for $F$. 
Recently, Zhang, Lin and Hou solved the existence problem for the case $\mu=0$ in the totally stable case [17] and the uni-instantaneous case [18]. They proved that if $m$ is a strictly positive, (almost-)invariant probability measure for $Q$, then there exists a $Q$-process $P$ for which $m$ is an invariant measure (and, hence, a stationary distribution). We will extend their results to the case $\mu>0$.

The structure of the paper is as follows. We begin, in Section 2, by examining the relationship between (6) and (7). Next, we recall the resolvent decomposition theorem of [2], which is the major tool for constructing uni-instantaneous $Q$-processes. This, and some other preliminary results, are presented in Section 3. Our main result on the existence of a $Q$-process with a given finite, almost- $\mu$-invariant measure for $Q$ is proved in Section 4. In Section 5, we discuss two examples illustrative of our results and, finally, in Section 6, we provide some necessary conditions for $\mu$-invariance. The terminology and notation used will follow that established by Anderson [1] and Yang [16].

\section{Almost $\mu$-invariance}

Our aim here is to provide necessary and sufficient conditions for a measure $m$ that satisfies (7) (but is not necessarily finite) to be almost $\mu$-invariant for $Q$. To do so, we recall the notions of an almost- $B$-type and an almost- $F$-type $Q$-process.

Definition 1. (Chen and Renshaw [3].) A uni-instantaneous $Q$-process $P$ with instantaneous state $b$ is called almost $B$-type if it satisfies the Kolmogorov backward equations over the noninstantaneous states, that is if

$$
p_{i j}^{\prime}(t)=\sum_{k \in S} q_{i k} p_{k j}(t), \quad i \neq b, j \in S
$$

The process $P$ is called almost $F$-type if it satisfies the Kolmogorov forward equations over the noninstantaneous states, that is if

$$
p_{i j}^{\prime}(t)=\sum_{k \in S} p_{i k}(t) q_{k j}, \quad i \neq b, j \in S
$$

By adapting the proof of Theorem 1 of [11], we can establish the following result.

Theorem 1. If $m$ is a $\mu$-invariant measure for $P$, then $m$ is almost $\mu$-invariant for $Q$ if and only if $P$ is almost F-type.

Proof. Since (7) holds, we may define an honest standard transition function $P^{*}(t)=$ $\left(p_{i j}^{*}(t), i, j \in S\right)$ over $S$ by

$$
p_{i j}^{*}(t)=\mathrm{e}^{\mu t} \frac{m_{j} p_{j i}(t)}{m_{i}}, \quad i, j \in S, t>0
$$

Indeed, $P^{*}$ is a $Q^{*}$-transition function, where $Q^{*}=\left(q_{i j}^{*}, i, j \in S\right)$ is the $q$-matrix with entries

$$
q_{i j}^{*}=\frac{m_{j} q_{j i}}{m_{i}}+\mu \delta_{i j}, \quad i, j \in S .
$$


( $P^{*}$ is called the $\mu$-reverse of $P$ with respect to $m$ and $Q^{*}$ the $\mu$-reverse of $Q$ with respect to $m$; see [9].) It is easy to see that $Q^{*}$ is uni-instantaneous with instantaneous state $b$ and, for $i \neq b$, that

$$
m_{i} \sum_{j \in S} q_{i j}^{*}=\sum_{j \neq i} m_{j} q_{j i}+\mu m_{i}-m_{i} q_{i} \leq 0 .
$$

Moreover, all of the states $i \neq b$ are conservative states for $Q^{*}$ if and only if (6) holds. It is easy to verify that $P^{*}$ is almost $B$-type if and only if $P$ is almost $F$-type. Thus, if (6) holds then $Q^{*}$ is conservative for the states $i \neq b$. Hence, the backward equations (8) hold for $P^{*}$ over the states $i \neq b$, implying that $P$ is almost $F$-type. Conversely, if $P$ is almost $F$-type then $P^{*}$ is almost $B$-type; however, $P^{*}$ is honest, implying that the states $i \neq b$ are conservative states for $Q^{*}$ and, hence, (6) holds.

\section{The resolvent decomposition theorem}

Henceforth, we will find it convenient to specify transition functions through their Laplace transforms. If $P$ is a specified transition function, then the function $\Psi(\alpha)=\left(\psi_{i j}(\alpha), i, j \in S\right)$ given by

$$
\psi_{i j}(\alpha)=\int_{0}^{\infty} \mathrm{e}^{-\alpha t} p_{i j}(t) \mathrm{d} t, \quad i, j \in S, \alpha>0,
$$

is called the resolvent of $P$. Indeed, if $i, j \in C$, where $C$ is any irreducible class, then the integral in (9) converges for all $\alpha>-\lambda_{P}(C)$, where $\lambda_{P}(C)$ is the decay parameter of $C$ (for $P$ ); see [6]. In analogy to properties (1)-(4) of $P$, the resolvent satisfies

$$
\begin{aligned}
& \psi_{i j}(\alpha) \geq 0, \quad i, j \in S, \alpha>0, \\
& \sum_{j \in S} \alpha \psi_{i j}(\alpha) \leq 1, \quad i \in S, \alpha>0, \\
& \psi_{i j}(\alpha)-\psi_{i j}(\beta)+(\alpha-\beta) \sum_{k \in S} \psi_{i k}(\alpha) \psi_{k j}(\beta)=0, \quad i, j \in S, \alpha, \beta>0, \\
& \lim _{\alpha \rightarrow \infty} \alpha \psi_{i j}(\alpha)=\delta_{i j}, \quad i, j \in S .
\end{aligned}
$$

(Note that (12) is called the resolvent equation.) Indeed, any $\Psi$ that satisfies (10)-(13) is the resolvent of a standard transition function $P$; see Lemma 1.1 of [12]. Furthermore, (11) is satisfied with equality if and only if $P$ is honest, in which case the resolvent is said to be honest. Also, the $q$-matrix of $P$ can be recovered from $\Psi$ using the following identity:

$$
q_{i j}=\lim _{\alpha \rightarrow \infty} \alpha\left(\alpha \psi_{i j}(\alpha)-\delta_{i j}\right)
$$

Finally, a resolvent $\Psi$ that satisfies (14) is called a $Q$-resolvent.

We can identify $\mu$-invariant measures using resolvents. If $P$ is a $Q$-process with resolvent $\Psi$ and $m=\left(m_{j}, j \in S\right)$ is a $\mu$-invariant measure for $P$, then $\mu \leq \lambda_{P}(S)$, where $\lambda_{P}(S)=$ $\inf _{C} \lambda_{P}(C)$ (the infimum being taken over all the irreducible classes comprising $S$ ); see Lemma 4.1 of [15]. Furthermore, since the integral in (9) converges for all $\alpha>-\lambda_{P}(S)$, we have

$$
\sum_{i \in S} m_{i} \alpha \psi_{i j}(\alpha-\mu)=m_{j}
$$


for all $j \in S$ and $\alpha>0$. We refer to $m$ as $\mu$-invariant for $\Psi$ if (15) is satisfied. Finally, a simple extension of Lemma 1 of [10] establishes both that $m$ is $\mu$-invariant for $\Psi$ if it is $\mu$-invariant for $P$, and that if $\mu \leq \lambda_{P}(S)$, then $m$ is $\mu$-invariant for $P$ if it is $\mu$-invariant for $\Psi$.

We are assuming that $Q$ is a uni-instantaneous $q$-matrix with instantaneous state $b$, so let us write $N=S \backslash\{b\}$ and denote by $Q_{N}=\left(q_{i j}, i, j \in N\right)$ the restriction of $Q$ to $N$. If $m=\left(m_{i}, i \in S\right)$ is a measure on $S$, then $m_{N}=\left(m_{i}, i \in N\right)$ will be the restriction of $m$ to $N$.

The following important result combines Theorems 7.7 and 7.8 of [2]. It characterizes $Q$-processes with a single instantaneous state. In preparation, define families $H_{\Psi}$ and $K_{\Psi}$, for a given $Q_{N}$-resolvent $\Psi(\alpha)=\left(\psi_{i j}(\alpha), i, j \in N\right)$, as follows: $H_{\Psi}$ is the set of all nonnegative row vectors $\eta(\alpha)=\left(\eta_{i}(\alpha), i \in N\right), \alpha>0$, satisfying $\sum_{j \in N} \eta_{j}(\alpha)<\infty$ and

$$
\eta_{j}(\alpha)-\eta_{j}(\beta)+(\alpha-\beta) \sum_{k \in N} \eta_{k}(\alpha) \psi_{k j}(\beta)=0, \quad j \in N
$$

and $K_{\Psi}$ is the set of all column vectors $\xi(\alpha)=\left(\xi_{i}(\alpha), i \in N\right), \alpha>0$, satisfying $0 \leq \xi_{i}(\alpha)$ $\leq 1, i \in N$, and

$$
\xi_{i}(\alpha)-\xi_{i}(\beta)+(\alpha-\beta) \sum_{k \in N} \psi_{i k}(\alpha) \xi_{k}(\beta)=0, \quad i \in N
$$

Theorem 2. (Resolvent decomposition theorem.) For the uni-instantaneous $q$-matrix $Q$, every $Q$-resolvent $R(\alpha)=\left(r_{i j}(\alpha), i, j \in S\right)$ can be decomposed uniquely as

$$
R(\alpha)=\left(\begin{array}{cc}
0 & 0 \\
0 & \psi(\alpha)
\end{array}\right)+r_{b b}(\alpha)\left(\begin{array}{cc}
1 & \eta(\alpha) \\
\xi(\alpha) & \xi(\alpha) \eta(\alpha)
\end{array}\right),
$$

where $\Psi(\alpha)=\left(\psi_{i j}(\alpha), i, j \in N\right)$ is a $Q_{N}$-resolvent and $\eta(\alpha)=\left(\eta_{i}(\alpha), i \in N\right)$ and $\xi(\alpha)=\left(\xi_{i}(\alpha), i \in N\right)$ satisfy the following conditions:

(i) $\eta(\alpha) \in H_{\Psi}$ and $\xi(\alpha) \in K_{\Psi}$,

(ii) $\xi_{i}(\alpha) \leq 1-\sum_{j \in N} \alpha \psi_{i j}(\alpha), i \in N$,

(iii) $\lim _{\alpha \rightarrow \infty} \alpha \eta_{j}(\alpha)=q_{b j}, j \in N$,

(iv) $\lim _{\alpha \rightarrow \infty} \alpha \xi_{i}(\alpha)=q_{i b}, i \in N$, and

(v) $r_{b b}(\alpha)=\left(C+\alpha+\alpha \sum_{j \in N} \eta_{j}(\alpha) \xi_{j}\right)^{-1}$, where $\xi_{j}:=\lim _{\alpha \rightarrow 0} \xi_{j}(\alpha)$ and $C<\infty$ satisfy

$$
\begin{gathered}
C \geq \lim _{\alpha \rightarrow \infty} \alpha \sum_{j \in N} \eta_{j}(\alpha)\left(1-\xi_{j}\right) \\
\lim _{\alpha \rightarrow \infty} \alpha \sum_{j \in N} \eta_{j}(\alpha) \xi_{j}=\infty \quad\left(\text { or, equivalently, } \quad \lim _{\alpha \rightarrow \infty} \alpha \sum_{j \in N} \eta_{j}(\alpha)=\infty\right) .
\end{gathered}
$$

Conversely, if there exists a $Q_{N}$-resolvent $\Psi$, and vectors $\eta(\alpha)$ and $\xi(\alpha)$ satisfying the above conditions, then $R$, defined by (17), is a $Q$-resolvent. 
Our main result rests on the following three lemmas.

Lemma 1. Suppose that the uni-instantaneous $q$-matrix $Q$ admits an almost- $\mu$-invariant measure $m=\left(m_{i}, i \in S\right)$. Then $d_{i}(\alpha)=\left(d_{i}(\alpha), i \in N\right)$, defined by

$$
d_{i}(\alpha)=m_{i}-(\alpha+\mu) \sum_{k \in N} m_{k} \phi_{k i}(\alpha), \quad i \in N, \alpha>0,
$$

where $\Phi_{N}(\alpha)=\left(\phi_{i j}(\alpha), i, j \in N\right)$ is the minimal $Q_{N}$-resolvent, satisfies

$$
\lim _{\alpha \rightarrow \infty} \alpha d_{i}(\alpha)=m_{b} q_{b i}, \quad i \in N .
$$

Proof. Since $m$ is almost $\mu$-invariant for $Q$, it is clear that the restriction $m_{N}=\left(m_{i}, i \in N\right)$ is a $\mu$-subinvariant measure for $Q_{N}$. Therefore, because $m_{N}$ is then $\mu$-subinvariant for $\Phi_{N}$, we find that $d_{i}(\alpha) \geq 0, i \in N, \alpha>0$. Also, since $\Phi_{N}$ is the minimal $Q_{N}$-resolvent, it satisfies the resolvent equation

$$
\phi_{i j}(\alpha)-\phi_{i j}(\beta)+(\alpha-\beta) \sum_{k \in N} \phi_{i k}(\alpha) \phi_{k j}(\beta)=0, \quad i, j \in N, \alpha, \beta>0,
$$

and, therefore,

$$
\bar{d}_{i}(\alpha)-\bar{d}_{i}(\beta)+(\alpha-\beta) \sum_{k \in N} \bar{d}_{k}(\alpha) \phi_{k j}(\beta)=0, \quad i \in N, \alpha, \beta>0,
$$

where

$$
\bar{d}_{i}(\alpha)=m_{i}-\alpha \sum_{k \in N} m_{k} \phi_{k i}(\alpha), \quad i \in N, \alpha>0 .
$$

Since $d_{i}(\alpha) \geq 0, i \in N, \alpha>0$, we have $\bar{d}_{i}(\alpha) \geq 0, i \in N, \alpha>0$. Using (20) we see that, for each $i \in N, \bar{d}_{i}(\alpha)$ is nonincreasing in $\alpha$ and, hence, $\alpha \sum_{k \in N} m_{k} \phi_{k i}(\alpha)$ is nondecreasing in $\alpha$. Therefore, $\lim _{\alpha \rightarrow \infty} \alpha \sum_{k \in N} m_{k} \phi_{k i}(\alpha)$ exists. However, by Fatou's lemma, $\lim _{\alpha \rightarrow \infty} \alpha \sum_{k \in N} m_{k} \phi_{k i}(\alpha) \geq m_{i}$, and, hence, $\lim _{\alpha \rightarrow \infty} \alpha \sum_{k \in N} m_{k} \phi_{k i}(\alpha)=m_{i}$ because $\bar{d}_{i}(\alpha) \geq 0$. Since $\Phi_{N}$ satisfies the forward equation

$$
\alpha \phi_{i j}(\alpha)=\delta_{i j}+\sum_{k \in N} \phi_{i k}(\alpha) q_{k j}, \quad i, j \in N, \alpha>0,
$$

and (19) can be rewritten as

$$
d_{i}(\alpha)=\sum_{k \in N} m_{k}\left(\delta_{k i}-(\alpha+\mu) \phi_{k i}(\alpha)\right), \quad i \in N, \alpha>0,
$$

we deduce that

$$
\begin{aligned}
\alpha d_{i}(\alpha) & =-\alpha \sum_{k \in N} m_{k} \sum_{j \in N} \phi_{k j}(\alpha) q_{j i}-\alpha \mu \sum_{k \in N} m_{k} \phi_{k i}(\alpha), \\
& =-\sum_{j \in N} q_{j i} \alpha \sum_{k \in N} m_{k} \phi_{k j}(\alpha)-\mu \alpha \sum_{k \in N} m_{k} \phi_{k i}(\alpha),
\end{aligned}
$$

which leads to

$$
\lim _{\alpha \rightarrow \infty} \alpha d_{i}(\alpha)=-\sum_{j \in N} m_{j} q_{j i}-\mu m_{i}=m_{b} q_{b i}, \quad i \in N
$$

This completes the proof. 
Lemma 2. Let $\Psi(\alpha)=\left(\psi_{i j}(\alpha), i, j \in N\right)$ be a $Q_{N}$-resolvent and let $\xi_{i}=\lim _{\alpha \rightarrow 0} \xi_{i}(\alpha)$, where $\xi_{i}(\alpha)=1-\alpha \sum_{j \in N} \psi_{i j}(\alpha), i \in N$. If $\eta(\alpha) \in H_{\Psi}$ then $\alpha \sum_{i \in N} \eta_{i}(\alpha)\left(1-\xi_{i}\right)$ is finite and does not depend on $\alpha$.

Proof. By the dominated convergence theorem,

$$
\begin{aligned}
\lim _{\beta \rightarrow 0} \alpha \beta \sum_{i \in N} \sum_{j \in N} \eta_{i}(\alpha) \psi_{i j}(\beta) & =\alpha \sum_{i \in N} \eta_{i}(\alpha) \lim _{\beta \rightarrow 0} \beta \sum_{j \in N} \psi_{i j}(\beta) \\
& =\alpha \sum_{i \in N} \eta_{i}(\alpha) \lim _{\beta \rightarrow 0}\left(1-\xi_{i}(\beta)\right) \\
& =\alpha \sum_{i \in N} \eta_{i}(\alpha)\left(1-\xi_{i}\right)
\end{aligned}
$$

On the other hand, using (16), we obtain

$$
\begin{aligned}
\lim _{\beta \rightarrow 0} \alpha \beta \sum_{i \in N} \sum_{j \in N} \eta_{i}(\alpha) \psi_{i j}(\beta) & =\lim _{\beta \rightarrow 0} \alpha \beta \sum_{j \in N} \sum_{i \in N} \eta_{i}(\alpha) \psi_{i j}(\beta) \\
& =\lim _{\beta \rightarrow 0} \frac{\alpha \beta}{\beta-\alpha} \sum_{j \in N}\left(\eta_{j}(\alpha)-\eta_{j}(\beta)\right) \\
& =\lim _{\beta \rightarrow 0} \frac{\alpha \beta}{\beta-\alpha} \sum_{j \in N} \eta_{j}(\alpha)+\lim _{\beta \rightarrow 0} \frac{\alpha \beta}{\alpha-\beta} \sum_{j \in N} \eta_{j}(\beta)
\end{aligned}
$$

The first term vanishes because $\sum_{j \in N} \eta_{j}(\alpha)<\infty$. The second term equals

$$
\lim _{\beta \rightarrow 0} \beta \sum_{j \in N} \eta_{j}(\beta)
$$

which exists, because it is easy to deduce, from (16), that $\beta \sum_{j \in N} \eta_{j}(\beta)$ is nondecreasing in $\beta$. Since this limit does not depend on $\alpha$, the proof is complete.

Lemma 3. Suppose that $m=\left(m_{i}, i \in S\right)$ is a strictly positive probability measure. If $m$ is $\mu$-invariant for the $Q$-resolvent $R$ defined in (17), then

(i) $m_{N}=\left(m_{i}, i \in N\right)$ is a $\mu$-subinvariant measure for $\Psi$, and

(ii) $\eta_{i}(\alpha)=d_{i}(\alpha) / m_{b}$, where $d_{i}(\alpha)=m_{i}-(\alpha+\mu) \sum_{k \in N} m_{k} \psi_{k i}(\alpha), i \in N, \alpha>0$.

Conversely, if (i) and (ii) hold, then, on setting $\xi_{i}(\alpha)=1-\alpha \sum_{j \in N} \psi_{i j}(\alpha), i \in N$, and $C=\mu / m_{b}+\alpha \sum_{i \in N} \eta_{i}(\alpha)\left(1-\xi_{i}\right)$, where $\xi_{i}=\lim _{\alpha \rightarrow 0} \xi_{i}(\alpha)$, (17) determines a $Q$-resolvent $R$ for which $m$ is a $\mu$-invariant measure.

Proof. If $m$ is $\mu$-invariant for $R$, that is

$$
(\alpha+\mu) \sum_{i \in S} m_{i} r_{i j}(\alpha)=m_{j}, \quad j \in S, \alpha>0,
$$


then $(\alpha+\mu) \sum_{i \in N} m_{i} \psi_{i j}(\alpha) \leq m_{j}, j \in N$, since, from (17), we have $\psi_{i j}(\alpha) \leq r_{i j}(\alpha)$, $i, j \in N$. This proves part (i). Next, from (17) and (21), we have

$$
(\alpha+\mu) r_{b b}(\alpha) m_{b}+(\alpha+\mu) \sum_{k \in N} m_{k} \xi_{k}(\alpha) r_{b b}(\alpha)=m_{b}
$$

and, for all $i \in N$ and $\alpha>0$,

$$
(\alpha+\mu) \eta_{i}(\alpha) r_{b b}(\alpha) m_{b}+(\alpha+\mu) \sum_{k \in N} m_{k} \psi_{k i}(\alpha)+(\alpha+\mu) \sum_{k \in N} m_{k} \xi_{k}(\alpha) r_{b b}(\alpha) \eta_{i}(\alpha)=m_{i}
$$

These equations combine to give $m_{b} \eta_{i}(\alpha)+(\alpha+\mu) \sum_{k \in N} m_{k} \psi_{k i}(\alpha)=m_{i}, i \in N$, and, hence, part (ii) holds.

To prove the converse, set $\xi_{i}(\alpha)=1-\alpha \sum_{j \in N} \psi_{i j}(\alpha)$ in (17) and take $\eta(\alpha)$ to satisfy (16). Then, by Lemma $2, \alpha \sum_{i \in N} \eta_{i}(\alpha)\left(1-\xi_{i}\right)$ is finite and independent of $\alpha$, and, so, the given $C$ satisfies (18). It follows that

$$
r_{b b}(\alpha)=\left(\frac{\mu}{m_{b}}+\alpha+\alpha \sum_{i \in N} \eta_{i}(\alpha)\right)^{-1}
$$

Since parts (i) and (ii) hold and $\sum_{i \in S} m_{i}=1$, we have

$$
\begin{aligned}
& (\alpha+\mu) r_{b b}(\alpha) m_{b}+(\alpha+\mu) \sum_{i \in N} m_{i} \xi_{i}(\alpha) r_{b b}(\alpha) \\
& \quad=r_{b b}(\alpha)\left((\alpha+\mu) m_{b}+(\alpha+\mu)\left(1-m_{b}\right)-\alpha \sum_{j \in N}(\alpha+\mu) \sum_{i \in N} m_{i} \psi_{i j}(\alpha)\right) \\
& \quad=r_{b b}(\alpha)\left(\mu+\alpha m_{b}+\alpha m_{b} \sum_{j \in N} \eta_{j}(\alpha)\right) \\
& \quad=m_{b}
\end{aligned}
$$

and, for $i \in N$,

$$
\begin{aligned}
& (\alpha+\mu) \eta_{i}(\alpha) r_{b b}(\alpha) m_{b}+(\alpha+\mu) \sum_{k \in N} m_{k} \psi_{k i}(\alpha)+(\alpha+\mu) \sum_{k \in N} m_{k} \xi_{k}(\alpha) r_{b b}(\alpha) \eta_{i}(\alpha) \\
& =(\alpha+\mu) r_{b b}(\alpha) d_{i}(\alpha)+(\alpha+\mu) \sum_{k \in N} m_{k} \psi_{k i}(\alpha)+(\alpha+\mu) r_{b b}(\alpha) \frac{d_{i}(\alpha)}{m_{b}} \sum_{k \in N} m_{k} \xi_{k}(\alpha) \\
& \quad=(\alpha+\mu) \sum_{k \in N} m_{k} \psi_{k i}(\alpha)+\frac{d_{i}(\alpha)}{m_{b}}\left((\alpha+\mu) r_{b b}(\alpha) m_{b}+(\alpha+\mu) \sum_{i \in N} m_{i} \xi_{i}(\alpha) r_{b b}(\alpha)\right) \\
& =(\alpha+\mu) \sum_{k \in N} m_{k} \psi_{k i}(\alpha)+d_{i}(\alpha) \\
& \quad=m_{i} .
\end{aligned}
$$

Thus, (22) and (23) hold. These in turn imply that (21) holds, meaning that $m$ is a $\mu$-invariant measure for $R$. 


\section{Existence}

We are now ready to state our main result.

Theorem 3. Let $\mu \geq 0$ and suppose that the uni-instantaneous $q$-matrix $Q$ admits a finite, almost- $\mu$-invariant measure $m=\left(m_{i}, i \in S\right)$. Then there exists a $Q$-process for which $m$ is a $\mu$-invariant measure.

Proof. Without loss of generality, we may assume that $\sum_{i \in S} m_{i}=1$. Let $\Phi(\alpha)=$ $\left(\phi_{i j}(\alpha), i, j \in N\right)$ be the minimal $Q_{N}$-resolvent. Since $m$ is almost $\mu$-invariant for $Q$, the restriction $m_{N}=\left(m_{i}, i \in N\right)$ is a $\mu$-subinvariant measure for $Q_{N}$ and, hence, is $\mu$-subinvariant for $\Phi$. Set

$$
\begin{array}{rlrl}
d_{i}(\alpha) & =m_{i}-(\alpha+\mu) \sum_{k \in N} m_{k} \phi_{k i}(\alpha), & & i \in N, \alpha>0, \\
\eta_{i}(\alpha)=\frac{d_{i}(\alpha)}{m_{b}}, & & i \in N, \alpha>0, \\
\xi_{i}(\alpha)=1-\alpha \sum_{j \in N} \phi_{i j}(\alpha), & & i \in N, \alpha>0,
\end{array}
$$

and

$$
r_{b b}(\alpha)=\left(\frac{\mu}{m_{b}}+\alpha+\alpha \sum_{i \in N} \eta_{i}(\alpha)\right)^{-1}
$$

Since $\Phi$ satisfies the resolvent equation, $\eta(\alpha)$ and $\xi(\alpha)$ given in (25) and (26) satisfy

$$
\eta_{i}(\alpha)-\eta_{i}(\beta)+(\alpha-\beta) \sum_{k \in N} \eta_{k}(\alpha) \phi_{k i}(\beta)=0, \quad i \in N
$$

and

$$
\xi_{i}(\alpha)-\xi_{i}(\beta)+(\alpha-\beta) \sum_{k \in N} \phi_{i k}(\alpha) \xi_{k}(\beta)=0, \quad i \in N .
$$

Using Lemma 1, we see that

$$
\lim _{\alpha \rightarrow \infty} \alpha \eta_{j}(\alpha)=\lim _{\alpha \rightarrow \infty} \alpha \frac{d_{j}(\alpha)}{m_{b}}=q_{b j}, \quad j \in N,
$$

and

$$
\lim _{\alpha \rightarrow \infty} \alpha \sum_{j \in N} \eta_{j}(\alpha)=\lim _{\alpha \rightarrow \infty} \frac{1}{m_{b}} \sum_{j \in N} \alpha d_{j}(\alpha)=\sum_{j \in N} q_{b j}=\infty
$$

Also,

$$
\lim _{\alpha \rightarrow \infty} \alpha \xi_{i}(\alpha)=\lim _{\alpha \rightarrow \infty} \sum_{k \in N} \alpha\left(\delta_{i k}-\alpha \phi_{i k}(\alpha)\right)=-\sum_{k \in N} q_{i k}=q_{i b}, \quad i \in N
$$


Therefore, using (26), (28), and Lemma 2, we deduce that $\alpha \sum_{i \in N} \eta_{i}(\alpha)\left(1-\xi_{i}\right)$ is finite and independent of $\alpha$. Now set

$$
C=\frac{\mu}{m_{b}}+\alpha \sum_{i \in N} \eta_{i}(\alpha)\left(1-\xi_{i}\right)
$$

where $\xi=\lim _{\alpha \rightarrow 0} \xi(\alpha)$, and observe that $C$ satisfies (18). Hence, in view of Theorem 2, we may use (24)-(27) to construct a $Q$-resolvent $R$ by setting

$$
R(\alpha)=\left(\begin{array}{cc}
0 & 0 \\
0 & \phi(\alpha)
\end{array}\right)+r_{b b}(\alpha)\left(\begin{array}{cc}
1 & \eta(\alpha) \\
\xi(\alpha) & \xi(\alpha) \eta(\alpha)
\end{array}\right)
$$

and then use the second part of Lemma 3 to deduce that $m$ is a $\mu$-invariant measure for $R$. This completes the proof.

Remark 1. When $\mu=0$, Theorem 3 reduces to the result of [18].

\section{Examples}

Example 1. We will begin with an example, generally known as the ' $\mathrm{K} 1$ ' chain, described by Kolmogorov [7] and analysed by Kendall and Reuter [5] and Reuter [13] (see also the discussions in [4] and [1]). The chain has a $q$-matrix over the nonnegative integers given by

$$
Q=\left(\begin{array}{ccccc}
-\infty & 1 & 1 & 1 & \ldots \\
q_{1} & -q_{1} & 0 & 0 & \ldots \\
q_{2} & 0 & -q_{2} & 0 & \ldots \\
q_{3} & 0 & 0 & -q_{3} & \ldots \\
\vdots & \vdots & \vdots & \vdots & \ddots
\end{array}\right)
$$

where $q_{i}>0, i \geq 1$. If a $\mu$-subinvariant measure exists for $Q$ then $\mu \leq \inf _{i} q_{i}$; see Corollary 1 of [6]. We will assume that $\mu<q_{i}$ for all $i \geq 1$. Then, for any such $\mu, Q$ admits a $\mu$-invariant measure $m=\left(m_{i}, i \geq 0\right)$ given by $m_{i}=m_{0} /\left(q_{i}-\mu\right), i \geq 1$, with $m_{0}$ arbitrary. This is finite if and only if

$$
\sum_{i=1}^{\infty} \frac{1}{q_{i}}<\infty
$$

in which case $Q$ has the unique $\mu$-invariant probability measure

$$
m_{0}=\frac{1}{A}, \quad m_{i}=\frac{m_{0}}{q_{i}-\mu}, \quad i \geq 1,
$$

where $A=1+\sum_{i=1}^{\infty} 1 /\left(q_{i}-\mu\right)$. Therefore, an immediate consequence of Theorems 2 and 3 and Lemma 3 is the following simple result.

Proposition 1. If $Q$ defined in (29) satisfies (30), then there exists a $Q$-process for which $m$, defined by (31), is a $\mu$-invariant probability measure. The resolvent of one such process is given by

$$
R(\alpha)=\left(\begin{array}{cc}
0 & 0 \\
0 & \phi(\alpha)
\end{array}\right)+r_{b b}(\alpha)\left(\begin{array}{cc}
1 & \eta(\alpha) \\
\xi(\alpha) & \xi(\alpha) \eta(\alpha)
\end{array}\right)
$$


where

$$
\begin{aligned}
\phi_{i j}(\alpha)=\frac{\delta_{i j}}{\alpha+q_{i}}, & i, j \geq 1, \alpha>0, \\
\xi_{i}(\alpha)=\frac{q_{i}}{\alpha+q_{i}}, & i \geq 1, \alpha>0, \\
\eta_{j}(\alpha)=\frac{1}{\alpha+q_{j}}, & j \geq 1, \alpha>0,
\end{aligned}
$$

and

$$
r_{b b}(\alpha)=\left(\frac{\mu}{m_{0}}+\alpha+\alpha \sum_{i=1}^{\infty} \eta_{i}(\alpha)\right)^{-1} .
$$

Example 2. Next we consider the following $q$-matrix, describing a birth-death process incorporating catastrophes to state 0 and instantaneous resurrection from state 0 :

$$
Q=\left(\begin{array}{ccccc}
-\infty & h_{1} & h_{2} & h_{3} & \ldots \\
d_{1} & -\left(d_{1}+b_{1}\right) & b_{1} & 0 & \ldots \\
d_{2} & a_{2} & -\left(a_{2}+b_{2}+d_{2}\right) & b_{2} & \ldots \\
d_{3} & 0 & a_{3} & -\left(a_{3}+b_{3}+d_{3}\right) & \ldots \\
\vdots & \vdots & \vdots & \vdots & \ddots
\end{array}\right) .
$$

Here, $d_{i}>0, b_{i}>0, i \geq 0, a_{i}>0, i \geq 1, h_{j} \geq 0, j \geq 1$, and $\sum_{j=1}^{\infty} h_{j}=\infty$. Define $\pi=\left(\pi_{i}, i \geq 1\right)$ by $\pi_{1}=1$ and

$$
\pi_{i}=\prod_{j=2}^{i} \frac{b_{j-1}}{a_{j}}, \quad i \geq 2 .
$$

It is easy to show that if $\mu$ satisfies $0 \leq \mu \leq \inf _{i \geq 1} d_{i}$ and if $h_{i}=c \pi_{i}\left(d_{i}-\mu\right), \quad i \geq 1$, where $c$ is a positive constant, then $m=\left(m_{i}, i \geq 0\right)$ given by

$$
m_{0}=1, \quad m_{i}=c \pi_{i}, \quad i \geq 1,
$$

is a $\mu$-invariant measure for $Q$.

Proposition 2. If $\mu$ satisfies $0 \leq \mu \leq \inf _{i \geq 1} d_{i}$ and $Q$ defined in (32) satisfies $\sum_{i=1}^{\infty} \pi_{i}<\infty$ and $\sum_{i=1}^{\infty} \pi_{i} d_{i}=\infty$, then there exists a $Q$-process for which $m$, defined by (33), is a $\mu$-invariant probability measure.

Proof. The condition $\sum_{i=1}^{\infty} \pi_{i}<\infty$ implies that $m$ is a finite measure, and the facts that $\sum_{i=1}^{\infty} \pi_{i} d_{i}=\infty$ and $\sum_{i=1}^{\infty} \pi_{i}<\infty$ together imply that $\sum_{j=1}^{\infty} h_{j}=\infty$. Hence, the result follows from Theorem 3 .

\section{Necessary conditions}

In both of the examples above, our finite measure $m$ satisfied

$$
\sum_{i \neq b} m_{i} q_{i b}=\infty
$$

and, hence, was invariant for $Q$ (that is, (5) holds for all $j \in S$ ). We have established that only almost $\mu$-invariance is needed for the existence of a $Q$-process for which the given (finite) 
measure is $\mu$-invariant. It would therefore be of interest to know whether (34) is actually necessary for a (finite or infinite) measure $m$ to be $\mu$-invariant for $P$. We shall content ourselves with the following result, which shows that (34) is necessary in the $\mu=0$ case under the condition that $P$ is reversible.

Theorem 4. Let $Q$ be a uni-instantaneous $q$-matrix with instantaneous state $b$ and let $P$ be $a$ $Q$-process with invariant measure $m$. If $P$ is reversible with respect to $m$, that is if

$$
m_{i} p_{i j}(t)=m_{j} p_{j i}(t), \quad i, j \in S,
$$

then (34) holds.

Proof. On dividing (35) by $t$ and letting $t \downarrow 0$, we obtain $m_{i} q_{i j}=m_{j} q_{j i}, j \neq i$. Hence,

$$
\sum_{i \neq j} m_{i} q_{i j}=m_{j} \sum_{i \neq j} q_{j i}, \quad j \in S,
$$

meaning that, in particular,

$$
\sum_{i \neq b} m_{i} q_{i b}=m_{b} \sum_{i \neq b} q_{b i}=\infty,
$$

since $Q$ is conservative.

We gain some insight into the general case from the following simple result, which follows directly from the proof of Theorem 1 .

Theorem 5. Let $Q$ be a uni-instantaneous $q$-matrix with instantaneous state $b$ and let $P$ be $a$ $Q$-process with $\mu$-invariant measure $m$. Let $P^{*}$ and $Q^{*}$ be, respectively, the $\mu$-reverse of $P$ with respect to $m$ and the $\mu$-reverse of $Q$ with respect to $m$. Then $P^{*}$ is honest. In particular, $b$ is an honest state for $P^{*}$, while being instantaneous for $Q^{*}$. Moreover,

$$
m_{b} \sum_{j \neq b} q_{b j}^{*}=\sum_{j \neq b} m_{j} q_{j b},
$$

meaning that, in particular, $b$ is a conservative state for $Q^{*}$ if and only if (34) holds.

\section{Acknowledgements}

We are grateful to the referee for detailed and thought-provoking comments and suggestions. The work of the first author was carried out during the period he spent as a vacation scholar in the Department of Mathematics of the University of Queensland. The support of the Australian Research Council (grant no. CE0348217) and the Australian Research Council Centre of Excellence for Mathematics and Statistics of Complex Systems is gratefully acknowledged.

\section{References}

[1] Anderson, W. J. (1991). Continuous-Time Markov Chains. An Applications-Oriented Approach. Springer, New York.

[2] Chen, A. Y. and Renshaw, E. (1990). Markov branching processes with instantaneous immigration. Prob. Theory Relat. Fields 87, 209-240.

[3] Chen, A. Y. and Renshaw, E. (1993). Existence and uniqueness criteria for conservative uni-instantaneous denumerable Markov processes. Prob. Theory Relat. Fields 94, 427-456. 
[4] Chung, K. (1967). Markov Chains with Stationary Transition Probabilities, 2nd edn. Springer, New York.

[5] Kendall, D. G. And Reuter, G. E. H. (1956). Some pathological Markov processes with a denumerable infinity of states and the associated semigroups of operators on l. In Proc. Internat. Congr. Math. 1954, Amsterdam, Vol. 3, Noordhoff, Groningen, pp. 377-415.

[6] Kingman, J. F. C. (1963). The exponential decay of Markov transition probabilities. Proc. London Math. Soc. 13, 337-358.

[7] Kolmogorov, A. N. (1951). On the differentiability of the transition probabilities in stationary Markov processes with a denumerable number of states. Moskov. Gos. Univ. Učenye Zapiski Mate. 148, 53-59 (in Russian).

[8] Pollett, P. K. (1986). On the equivalence of $\mu$-invariant measures for the minimal process and its $q$-matrix. Stoch. Process. Appl. 22, 203-221.

[9] Pollett, P. K. (1988). Reversibility, invariance and $\mu$-invariance. Adv. Appl. Prob. 20, 600-621.

[10] Pollett, P. K. (1991). On the construction problem for single-exit Markov chains. Bull. Austral. Math. Soc. 43, 439-450.

[11] Pollett, P. K. and Vere-Jones, D. (1992). A note on evanescent processes. Austral. J. Statist. 34, 531-536.

[12] Reuter, G. E. H. (1959). Denumerable Markov processes. II. J. London Math. Soc. 34, 81-91.

[13] Reuter, G. E. H. (1969). Remarks on a Markov chain example of Kolmogorov. Z. Wahrscheinlichkeitsth. 13, 315-320.

[14] TweEdiE, R. L. (1974). Some ergodic properties of the Feller minimal process. Quart. J. Math. Oxford Ser. 25, 485-495.

[15] Vere-Jones, D. (1967). Ergodic properties of nonnegative matrices. I. Pacific J. Math. 22, 361-386.

[16] Yang, X. Q. (1990). The Construction Theory of Denumerable Markov Processes. John Wiley, Chichester.

[17] Zhang, H. J., Lin, X. And Hou, Z. T. (2001). Invariant distributions of $Q$-processes. I. Chinese Ann. Math. Ser. A 22, 323-330.

[18] Zhang, H. J., Lin, X. And Hou, Z. T. (2002). Invariant distributions of $Q$-processes. II. Chinese Ann. Math. Ser. A 23, 361-370. 